\title{
A narrative review of chimeric antigen receptor-T (CAR-T) cell therapy for lung cancer
}

\author{
Yujia Liu $^{1 \wedge}$, Yayi He ${ }^{1,2}$ \\ ${ }^{1}$ Tongji University, Shanghai, China; ${ }^{2}$ Department of Medical Oncology, Shanghai Pulmonary Hospital, Tongji University Medical School Cancer \\ Institute, Tongji University School of Medicine, Shanghai, China \\ Contributions: (I) Conception and design: Y He; (II) Administrative support: Y He; (III) Provision of study materials or patients: Y Liu; (IV) Collection \\ and assembly of data: Y Liu; (V) Data analysis and interpretation: Y Liu; (VI) Manuscript writing: Both authors; (VII) Final approval of manuscript: \\ Both authors. \\ Correspondence to: Yayi He. No. 507 Zhengmin Road, Shanghai 200433, China. Email: 2250601@qq.com.
}

\begin{abstract}
Lung cancer represents one of the most common and deadliest cancers in the world. Chimeric antigen receptor- $\mathrm{T}$ cell (CAR-T) therapy which can recognize antigens in a major histocompatibility complex (MHC)-independent manner provides a new approach for tumor treatment. However, lung cancer, as a solid tumor, faces several formidable barriers to adoptive cell transfer, which includes inhibition of T-cell localization and suppression of T-cell function. Therefore, lung cancer fails to respond significantly to infusions of CAR-T cells in most trials until now. PubMed was researched using the terms "CAR-T" and "lung cancer" only in English from 2000 through June 2020. We also included results presented in international conferences, such as the American Society of Clinical Oncology (ASCO) and the European Society for Medical Oncology (ESMO). Besides, we found new progress in CAR-T therapy for solid tumors as a supplement. To enhance the efficacy and conquer the limitations, we collected some applications in lung cancer. In recent years, there have been some improvements in selecting the proper target and reducing toxicity. CAR-T technology provides an excellent way for tumor treatment, which does not depend on MHC molecules and provides a new method for the utilization of tumor targets. Targeting different antigens and overcoming the solid barrier, there are some improvements in responding significantly and reducing toxicity. CAR-T technology will play a decisive role in the treatment of lung cancer.
\end{abstract}

Keywords: Chimeric antigen receptor-T (CAR-T); lung cancer; cancer immunity

Submitted Jul 20, 2020. Accepted for publication Mar 14, 2021.

doi: $10.21037 / \mathrm{atm}-20-5419$

View this article at: http://dx.doi.org/10.21037/atm-20-5419

\section{Introduction}

\section{Background}

Lung cancer represents one of the most common and deadliest cancers in the world. Siegel et al. estimated that 606,880 Americans will die from cancer in 2019 and onequarter of the deaths are from lung cancer both in men and women (1). Despite surgical resection and chemotherapy and radiation, advances in survival have been slow for lung cancer (1). Recently, immunotherapy drugs such as programmed cell death 1 inhibitor (PD-1) have been approved to treat non-small cell lung carcinoma (NSCLC) as well as in combination with chemotherapy for small cell lung carcinoma (SCLC) (2).

Chimeric antigen receptor-T (CAR-T) cell therapy which can recognize antigens in a major histocompatibility complex (MHC)-independent manner is a hot topic in the field, providing a new approach for tumor treatment.

^ ORCID: 0000-0002-9810-518X. 


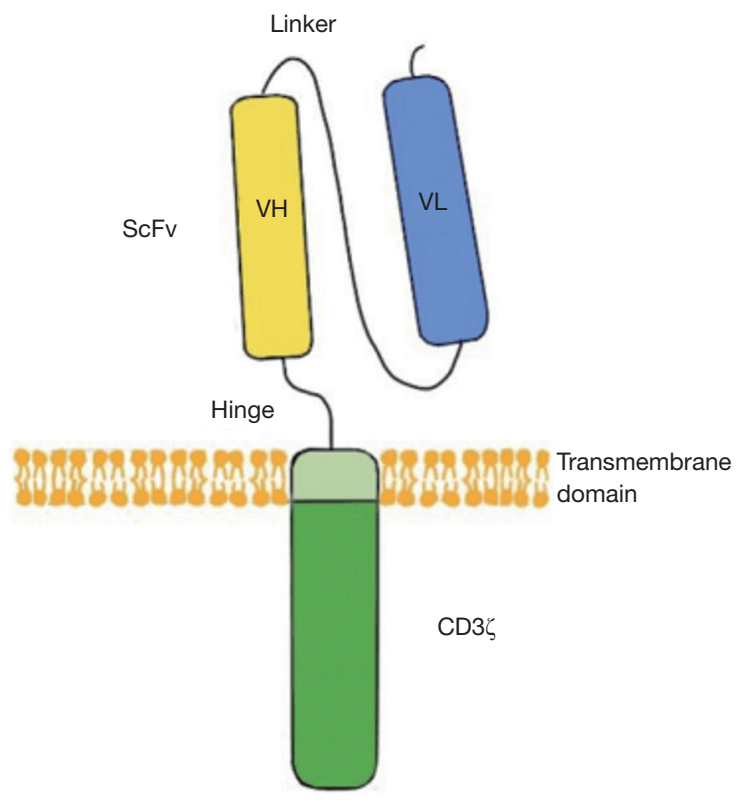

Figure 1 The structure of CAR. CARs are comprised of three parts: scFv located extracellularly to recognize antigen, a transmembrane domain, and $\mathrm{CD} 3 \zeta$ which is intracellular and can active $\mathrm{T}$ cell. $\mathrm{scFv}$, the single-chain fragment variant; $\mathrm{CD} 3 \zeta$, cluster of differentiation $3 \zeta$; $\mathrm{VH}$, the variable heavy; $\mathrm{VL}$, the variable heavy light.

However, solid tumors face several formidable barriers to adoptive cell transfer, which includes inhibition of T-cell localization and suppression of T-cell function. Therefore, lung cancer fails to respond significantly to infusions of CAR-T cells in most trials until now.

\section{Engineering CAR-T}

CARs are engineered receptors inserting a discretional specificity onto a $\mathrm{T}$ cell. CARs are comprised of three parts: the single-chain fragment variant $(\mathrm{scFv})$ located extracellularly to recognize antigen, a transmembrane domain, and $\mathrm{CD} 3 \zeta$ which is intracellular and can active $\mathrm{T}$ cell (Figure 1). CAR-T therapy would redirect the $\mathrm{T}$ cells to a specific target to attack tumor cells. This approach without MHC restriction works well, especially for hematologic malignancies (3).

According to the structure of the endo-domain CAR-T and cancer immunity, CAR-T can be divided into four generations. For the first generation, $\mathrm{CD} 3 \zeta$ or other singlechain antibodies would link the ITAM (immunoreceptor tyrosine-based activation motif) at the transmembrane region. However, these CAR-T cells don't produce enough interleukin-2 (IL-2) to kill tumor cells and have low expression levels in vitro. So, it is necessary to provide exogenous IL-2. For the second generation, a costimulatory molecule (CM1) like CD28 is designed for the signal transduction region, providing additional signals to the $\mathrm{T}$ cell. CM1 can improve the proliferation, cytotoxicity, and sustained response, and prolong the life of CAR-T cells in vivo. As for the third generation, another costimulatory molecule (CM2) such as CD134 is engineered to the signal transduction region based on the second generation, augmenting potency with stronger cytokine production and killing ability. Again, developed from the second generation, the interleukin-12 (IL-12) is designed to the signal transduction region in the fourth generation (4). The fourth-generation CARs are known as T cells redirected for universal cytokine-mediated killing (TRUCKs). TRUCKs increase T-cell activation and attract innate immune cells to eliminate antigen-negative cancer cells in the targeted lesion. Such TRUCK-T cells can also treat viral infections, metabolic disorders, and auto-immune diseases.

There are two approaches in accomplishing gene incorporation with vectors: viral and non-viral systems. Non-viral gene therapy is widely used for cancer treatment because of its target specificity, high efficiency, unlimited carrier capacity, non-infectiousness, and controlled chemical constitution (4).

\section{CAR-T and cancer immunity}

CAR binds $T$ cells to the surface antigens of target cells through an scFv recognition domain and thus is MHCunrestricted. Forming a non-classical immune synapse (IS), CAR-T mediates the anti-tumor function through the granzyme and perforin, releasing the cytokines to sensitize the tumor stroma. The function and persistence in the host are closely related to the receptor's sections- $\mathrm{scFv}$, costimulatory domains, and spacer domain (5).

There are multiple hurdles in CAR-T therapy for lung cancer and other solid tumors. First, solid tumors would secrete chemokines such as CXCL12 (a gene on chromosome 10q11.1 that encodes a stromal cell-derived alpha chemokine of the intracrine family) to inhibit T-cell migration (6). Besides, the chemokine receptors on $\mathrm{T}$ cells often fail to match the signature of tumor cells, resulting in less migration to the tumor area (7). Second, the tumor microenvironment (TME) presents a physical barrier, inhibiting efficient infiltration and attracting the immune- 
suppressive myeloid cells (8). Third, the TME is crowded with multiple cellular and molecular components which would decrease the anti-tumor immune function (9).

We present the following article in accordance with the Narrative Review reporting checklist (available at http:// dx.doi.org/10.21037/atm-20-5419).

\section{Methods}

PubMed was researched using the terms "CAR-T" and "lung cancer" only in English from 2000 through June 2020. We also included results presented in international conferences, such as the American Society of Clinical Oncology (ASCO) and the European Society for Medical Oncology (ESMO). Besides, we found new progress in CAR-T therapy for solid tumors as a supplement. After that, the engineering of CAR-T and its relative cancer immunity were detailed and more experiments were cited to introduce the application in lung cancer. Then we talked about the limitations and tried to propose new ideas to improve this technology.

\section{Results}

\section{Application in lung cancer}

Unlike hematological malignancies, lung cancer shows heterogeneity of tumor antigens, has difficulty penetrating, and faces physical immunosuppressive factors.

Concerning choice for targets, some differentiation antigens are popular due to over-expression on cancer cells while low-expression on normal tissues. They can also be used as biomarkers, serving as prognostic markers. Mesothelin (MSLN) is one such antigen that is at a high level in the majority of lung cancer (10). Carcinoma embryonic antigen (CEA) which presents highly in lung cancer is an attractive marker to monitor the response to treatment and brain metastasis in advanced NSCLC (11). Human epidermal growth factor receptor-2 (HER2) overexpression has also been found in NSCLC and is proved concerning poor prognosis (12). Tumor-associated antigens (TAAs) such as epidermal growth factor receptor (EGFR) emerge as an important subtype of lung cancer, with an estimated prevalence of $10-15 \%$ in non-squamous NSCLC (13). Altered gene products from post-translational modifications such as mucin 1 (MUC1) are potential targeting candidates. The tumor-selective glycol-epitopes from the abnormal glycosylation relate to tumor progression and poor prognosis (14). Chinnasamy et al. have presented a new strategy by targeting and destroying the vasculature which could reduce blood flow and nutrient supplies to the tumor, restricting its development and enhancing T-cell infiltration (15). Hu et al. constructed a CAR-T-cell-based strategy to target lung-specific $\mathrm{X}$ (LunX). In vitro, CARLunX-T cells enhanced toxicity toward NSCLC and recognized specifically. They also constructed a patientderived xenograft model of lung cancer, finding that the survival was prolonged significantly (16). Kawaguchi et al. Prostate stem cell antigen (PSCA) is highly expressed in NSCLC and may be functionally important (17). The expression of receptor-tyrosine-kinase like orphan receptor 1 (ROR1) was significantly higher in lung adenocarcinoma (ADC) tissues than that in their adjacent non-tumor tissues, correlating with malignant attributes of lung ADC. ROR1 may serve as a novel prognostic marker (18). Although the clinical trials involving CAR-T therapy directed against lung cancer are still underway, researchers involved have disclosed some good results, showing considerable effect with acceptable toxicity (ASCO, 2019) (Table 1).

To overcome the solid barrier, a successful match between chemokine receptors and adhesion molecules on the $\mathrm{T}$ cells with those secreted and expressed by the tumor cells is necessary. Genetically modifying CAR-T to express the appropriate chemokine receptors like CXCR2 has been observed to migrate towards tumor cells expressing CXCL1 (19). Besides, there are studies to modulate chemokine secretion from tumors in other solid tumors (20).

There are some techniques to increase the penetration of CAR-T cells. Heparanase (HPSE) could degrade heparin sulfate proteoglycans which consist of the extracellular matrix (ECM) and thus promotes tumor infiltration and antitumor activity (21). Therefore, we could engineer CAR-T cells to express HPSE. Some researchers proposed to transport CAR-T cells entirely bypass the systemic circulation (22).

Immunosuppressive factors including checkpoint pathways, cytokines, and by-products from an altered metabolism could be blocked to enhance anti-tumor efficacy. For example, co-expression of IL-12 on CAR-T was demonstrated to repolarize the tumor microenvironment and cause tumor regression without exogenous IL-2 (23). John et al. found significant improvement for CAR-T therapy when used together with monoclonal antibodies against checkpoint molecules such as. anti-PD-1 antibody (24). Incorporating mutant or nullified cell-surface dominantnegative receptors (DNRs) to CAR-T cells could counteract the inactivating signals in the TME (25). Switch receptors 
Table 1 List of clinical trials involving CAR-T therapy directed against lung cancer

\begin{tabular}{|c|c|c|c|c|c|}
\hline Antigen & Type of cancer & Phase & ID & Cited in (PMID) & Outcomes \\
\hline EGFR & Lung, colorectal, ovary, pancreatic & 1 and 2 & NCT01869166 & 26968708,26574053 & No results posted \\
\hline GPC3 & Lung squamous cell carcinoma & 1 & NCT02876978 & - & No results posted \\
\hline MUC1 & HCC, NSCLC, pancreatic & 1 and 2 & NCT02587689 & 27550819 & No results posted \\
\hline
\end{tabular}

Trial information can be located using a trial ID at https://clinicaltrials.gov. CEA, carcinoembryonic antigen; EGFR, epidermal growth factor receptor; GPC3, glypican-3; HCC, hepatocellular carcinoma; HER2, human epidermal growth factor receptor 2; MUC1, mucin 1; NSCLC, non-small cell lung cancer.

such as PD-1-CD28 provide another alternative approach to avoid immunosuppression (26).

\section{Discussion}

\section{Limitations}

However, most antigens targeted by CAR-T are not "tumor-specific" but "tumor-associated". Thus, potential off-target toxicities present a big problem.

There are also some reports about immune-related adverse events (IRAEs), attenuating tolerance and causing overwhelming inflammation, autoimmunity, and tissue damage (27). Lynn found that c-Jun over-expression in CAR-T induces exhaustion resistance (28).

Cytokine-release syndrome (CRS) is a fatal systemic inflammatory reaction after the infusion of CAR-T, which includes fever, hypotension, skin reactions, and lab abnormalities. Inflammatory cytokines such as IL-6 are released after the activation of CAR-T and cytotoxic damage of macrophages, monocytes, and lymphocyte populations.

Besides CRS, treatment with CAR-T may also cause central nervous system (CNS) toxicity (29). Some patients develop reversible neurologic complications such as delirium and seizure-like symptom. There can also be a gradual progression of confusion, aphasia, and ultimately dementia. Some cases even required air protection such as intubation and mechanical ventilation (30).

The leading indications for intensive care units (ICU) admissions of cancer patients are dyspnea and hypoxemia. Besides, doctors should be aware of cardiovascular events.

\section{Prospective}

There are several approaches to improve the safety of selection for targets. First, Johnson et al. investigated that high-reactive CARs would recognize tumor antigens efficiently (31). Second, incorporating the suicide gene within these $\mathrm{T}$ cells would strengthen safety control. The iCasp9 cell-suicide system is reported to enhance the safety and widen the clinical applications (32). Researchers with the strategy to separate the signaling domains into two CARs; one contains only the costimulatory signaling domains such as CD28, while another with different specificity contains CD3z (33). Wilkie et al. found that T-cells with "dual-targeted" would kill ErbB2(+) tumor cells effectively and proliferate when target cells express both MUC1 and ErbB2 (34). AUTO3, the cell product targeting both CD19 and CD22, shows a lower affinity and a fast off rate, leading to enhanced activity and lower toxicities (ESMO 2020). What's more, injections of CAR-T electroporated with mRNAs behave safer and the toxicity would decrease rapidly. Therefore, RNA CAR-T is expected to be safer (35).

Parente-Pereira et al. found that human T cells would reach the lungs at first and remain for more than 3 hours. Then $\mathrm{T}$ cells redistribute to other organs like the liver, spleen, and lymph nodes (36). Delayed clearance of T cells during the first passthrough in the lungs is considered to be highly associated with pulmonary toxicity (37). To prevent severe toxicity such as CRS, a restricted doseescalation scheme of CAR-T should be cogitated in clinical applications (38). Integration of the cytoplasmic domain of CD $3 \varepsilon$ with a second-generation CAR could active the anti- 
tumor effect of CAR-T cells. It is revealed that we could reduce cytokine production of CAR by Csk-recruiting ITAM of CD3 $\varepsilon$ and produce CAR-T persistence via $\mathrm{p} 85$ recruitment of the basic residue rich sequence (BRS) of CD3 (39).

Similarly, bispecific killer cell engagers (BiKEs) are small molecules consisting of two scFvs, one targeting a tumor antigen (e.g., CD19, CD20, CD33), while the other specific for an NK cell receptor (CD16). BiKEs facilitates the formation of an immunological synapse, allowing NK cells to specifically and effectively execute cytolytic functions. Additional scFvs, such as tri-specific killer cell engagers (TriKEs), can further potentiate therapeutic benefits by targeting more tumor antigens or adding IL-15 into the engager construct (40). In a nutshell, BiKEs and TriKEs provide a non-cell-based immunotherapeutic approach that can harness the patients' own NK cells against cancer.

\section{Summary}

Lung cancer is heterogeneous in tumor antigens and its treatment needs to overcome solid barriers and physical immunosuppressive factors. There are some studies to provide enlightening approaches to solve these hurdles but still need more research. Moreover, its limitations and side effects are waiting to be solved. Despite these problems, we should be optimistic that the development of CAR-T technology provides an excellent way for tumor treatment, which does not depend on MHC molecules and provides a new method for the utilization of tumor targets. Through targeting different antigens and overcoming the solid barrier, there are some improvements in responding significantly and reducing toxicity. CAR-T technology will play a decisive role in the treatment of lung cancer.

\section{Acknowledgments}

Funding: This study was supported in part by a grant of National Natural Science Foundation of China (81802255), Clinical research project of Shanghai Pulmonary Hospital (FKLY20010), Young Talents in Shanghai (2019 QNBJ), Dream Tutor Outstanding Young Talents Program (fkyq1901), Clinical Research Project of Shanghai Pulmonary Hospital (fk18005), Key Discipline in 2019 (oncology), Project of Shanghai Municipal Science and Technology Commission (Project of Municipal Science and Technology Commission), Scientific research project of Shanghai Pulmonary Hospital (fkcx1903), Shanghai
Municipal Commission of Health and Family Planning (2017YQ050), Innovation Training Project of SITP of Tongji University, and key projects of leading talent (19411950300). Youth project of hospital management research fund of Shanghai Hospital Association (Q1902037).

\section{Footnote}

Reporting Checklist: The authors have completed the Narrative Review reporting checklist. Available at http:// dx.doi.org/10.21037/atm-20-5419

Conflicts of Interest: Both authors have completed the ICMJE uniform disclosure form (available at http://dx.doi. org/10.21037/atm-20-5419). The authors have no conflicts of interest to declare.

Ethical Statement: The authors are accountable for all aspects of the work in ensuring that questions related to the accuracy or integrity of any part of the work are appropriately investigated and resolved.

Open Access Statement: This is an Open Access article distributed in accordance with the Creative Commons Attribution-NonCommercial-NoDerivs 4.0 International License (CC BY-NC-ND 4.0), which permits the noncommercial replication and distribution of the article with the strict proviso that no changes or edits are made and the original work is properly cited (including links to both the formal publication through the relevant DOI and the license). See: https://creativecommons.org/licenses/by-nc-nd/4.0/.

\section{References}

1. Siegel RL, Miller KD, Jemal A. Cancer statistics, 2019. CA Cancer J Clin 2019;69:7-34.

2. Miller KD, Nogueira L, Mariotto AB, et al. Cancer treatment and survivorship statistics, 2019. CA Cancer J Clin 2019;69:363-85.

3. Lipowska-Bhalla G, Gilham DE, Hawkins RE, et al. Targeted immunotherapy of cancer with CAR T cells: achievements and challenges. Cancer Immunol Immunother 2012;61:953-62.

4. Zhang C, Liu J, Zhong JF, et al. Engineering CAR-T cells. Biomark Res 2017;5:22.

5. Benmebarek MR, Karches CH, Cadilha BL, et al. Killing Mechanisms of Chimeric Antigen Receptor (CAR) T Cells. Int J Mol Sci 2019;20:1283. 
6. Feig C, Jones JO, Kraman M, et al. Targeting CXCL12 from FAP-expressing carcinoma-associated fibroblasts synergizes with anti-PD-L1 immunotherapy in pancreatic cancer. Proc Natl Acad Sci U S A 2013;110:20212-7.

7. Harlin H, Meng Y, Peterson AC, et al. Chemokine expression in melanoma metastases associated with CD8+ T-cell recruitment. Cancer Res 2009;69:3077-85.

8. Tumeh PC, Harview CL, Yearley JH, et al. PD-1 blockade induces responses by inhibiting adaptive immune resistance. Nature 2014;515:568-71.

9. Yong CSM, Dardalhon V, Devaud C, et al. CAR T-cell therapy of solid tumors. Immunol Cell Biol 2017;95:356-63.

10. Morello A, Sadelain M, Adusumilli PS. MesothelinTargeted CARs: Driving T Cells to Solid Tumors. Cancer Discov 2016;6:133-46.

11. Lee DS, Kim YS, Jung SL, et al. The relevance of serum carcinoembryonic antigen as an indicator of brain metastasis detection in advanced non-small cell lung cancer. Tumour Biol 2012;33:1065-73.

12. Ricciardi GR, Russo A, Franchina T, et al. NSCLC and HER2: between lights and shadows. J Thorac Oncol 2014;9:1750-62.

13. Greenhalgh J, Dwan K, Boland A, et al. Firstline treatment of advanced epidermal growth factor receptor (EGFR) mutation positive non-squamous nonsmall cell lung cancer. Cochrane Database Syst Rev 2016;(5):CD010383.

14. Wilkie S, Picco G, Foster J, et al. Retargeting of human T cells to tumor-associated MUC1: the evolution of a chimeric antigen receptor. J Immunol 2008;180:4901-9.

15. Chinnasamy D, Tran E, Yu Z, et al. Simultaneous targeting of tumor antigens and the tumor vasculature using $T$ lymphocyte transfer synergize to induce regression of established tumors in mice. Cancer Res 2013;73:3371-80.

16. Hu Z, Zheng X, Jiao D, et al. LunX-CAR T Cells as a Targeted Therapy for Non-Small Cell Lung Cancer. Mol Ther Oncolytics 2020;17:361-70.

17. Kawaguchi T, Sho M, Tojo T, et al. Clinical significance of prostate stem cell antigen expression in non-small cell lung cancer. Jpn J Clin Oncol 2010;40:319-26.

18. Zheng YZ, Ma R, Zhou JK, et al. ROR1 is a novel prognostic biomarker in patients with lung adenocarcinoma. Sci Rep 2016;6:36447.

19. Kershaw MH, Wang G, Westwood JA, et al. Redirecting migration of $\mathrm{T}$ cells to chemokine secreted from tumors by genetic modification with CXCR2. Hum Gene Ther 2002;13:1971-80.
20. Nishio N, Diaconu I, Liu H, et al. Armed oncolytic virus enhances immune functions of chimeric antigen receptor-modified $\mathrm{T}$ cells in solid tumors. Cancer Res 2014;74:5195-205.

21. Caruana I, Savoldo B, Hoyos V, et al. Heparanase promotes tumor infiltration and antitumor activity of CAR-redirected T lymphocytes. Nat Med 2015;21:524-9.

22. Zeltsman M, Dozier J, McGee E, et al. CAR T-cell therapy for lung cancer and malignant pleural mesothelioma. Transl Res 2017;187:1-10.

23. Chinnasamy D, Yu Z, Kerkar SP, et al. Local delivery of interleukin-12 using T cells targeting VEGF receptor-2 eradicates multiple vascularized tumors in mice. Clin Cancer Res 2012;18:1672-83.

24. John LB, Devaud C, Duong CP, et al. Anti-PD-1 antibody therapy potently enhances the eradication of established tumors by gene-modified T cells. Clin Cancer Res 2013;19:5636-46.

25. Sheppard D. Dominant negative mutants: tools for the study of protein function in vitro and in vivo. Am J Respir Cell Mol Biol 1994;11:1-6.

26. Liu X, Ranganathan R, Jiang S, et al. A Chimeric SwitchReceptor Targeting PD1 Augments the Efficacy of Second-Generation CAR T Cells in Advanced Solid Tumors. Cancer Res 2016;76:1578-90.

27. Horvat TZ, Adel NG, Dang TO, et al. Immune-Related Adverse Events, Need for Systemic Immunosuppression, and Effects on Survival and Time to Treatment Failure in Patients With Melanoma Treated With Ipilimumab at Memorial Sloan Kettering Cancer Center. J Clin Oncol 2015;33:3193-8.

28. Lynn RC, Weber EW, Sotillo E, et al. c-Jun overexpression in CAR T cells induces exhaustion resistance. Nature 2019;576:293-300.

29. Kroschinsky F, Stölzel F, von Bonin S, et al. New drugs, new toxicities: severe side effects of modern targeted and immunotherapy of cancer and their management. Crit Care 2017;21:89.

30. Davila ML, Riviere I, Wang X, et al. Efficacy and toxicity management of $19-28 z$ CAR T cell therapy in B cell acute lymphoblastic leukemia. Sci Transl Med 2014;6:224ra25.

31. Johnson LA, Morgan RA, Dudley ME, et al. Gene therapy with human and mouse $\mathrm{T}$-cell receptors mediates cancer regression and targets normal tissues expressing cognate antigen. Blood 2009;114:535-46.

32. Di Stasi A, Tey SK, Dotti G, et al. Inducible apoptosis as a safety switch for adoptive cell therapy. N Engl J Med 2011;365:1673-83. 
33. Varela-Rohena A, Carpenito C, Perez EE, et al. Genetic engineering of $\mathrm{T}$ cells for adoptive immunotherapy. Immunol Res 2008;42:166-81.

34. Wilkie S, van Schalkwyk MC, Hobbs S, et al. Dual targeting of ErbB2 and MUC1 in breast cancer using chimeric antigen receptors engineered to provide complementary signaling. J Clin Immunol 2012;32:1059-70.

35. Zhao Y, Moon E, Carpenito C, et al. Multiple injections of electroporated autologous $\mathrm{T}$ cells expressing a chimeric antigen receptor mediate regression of human disseminated tumor. Cancer Res 2010;70:9053-61.

36. Parente-Pereira AC, Burnet J, Ellison D, et al. Trafficking of CAR-engineered human $\mathrm{T}$ cells following regional or systemic adoptive transfer in SCID beige mice. J Clin

Cite this article as: Liu Y, He Y. A narrative review of chimeric antigen receptor-T (CAR-T) cell therapy for lung cancer. Ann Transl Med 2021;9(9):808. doi: 10.21037/atm-20-5419
Immunol 2011;31:710-8.

37. Morgan RA, Yang JC, Kitano M, et al. Case report of a serious adverse event following the administration of $\mathrm{T}$ cells transduced with a chimeric antigen receptor recognizing ERBB2. Mol Ther 2010;18:843-51.

38. Shi H, Liu L, Wang Z. Improving the efficacy and safety of engineered $\mathrm{T}$ cell therapy for cancer. Cancer Lett 2013;328:191-7.

39. Wu W, Zhou Q, Masubuchi T, et al. Multiple Signaling Roles of CD $3 \varepsilon$ and Its Application in CAR-T Cell Therapy. Cell 2020;182:855-871.e23.

40. Felices M, Lenvik TR, Davis ZB, et al. Generation of BiKEs and TriKEs to Improve NK Cell-Mediated Targeting of Tumor Cells. Methods Mol Biol 2016;1441:333-46. 